\title{
Proposal of Sphingomonas wittichii sp. nov. for strain RW1T, known as a dibenzo-p-dioxin metabolizer
}

\author{
Eiko Yabuuchi, ${ }^{1}$ Hiroyuki Yamamoto, ${ }^{2}$ Shigemi Terakubo, ${ }^{2}$ \\ Ninyo Okamura, ${ }^{2}$ Takashi Naka, ${ }^{3,6}$ Nagatoshi Fujiwara, ${ }^{3}$ \\ Kazuo Kobayashi, ${ }^{3}$ Yoshimasa Kosako ${ }^{4}$ and Akira Hiraishi ${ }^{5}$
}

Author for correspondence: Eiko Yabuuchi. Tel: +8166951 1715. Fax: +81669563643. e-mail: achei-08@fa2.so-net.ne.jp

\begin{abstract}
A polyphasic taxonomic study was performed on Sphingomonas sp. strain RW1'. The organism was isolated from water of the River Elbe and has been known as a potent metabolizer of dibenzo-p-dioxin and its relatives. TLC of a mild alkaline hydrolysate of extractable cellular lipids of strain RW1' and type strains of 21 Sphingomonas species gave a spot of sphingoglycolipid (SGL)-1 (glucuronosyl ceramide), which is characteristic of sphingomonads. In addition, strain RW1 ${ }^{\top}$ and type strains of three Sphingomonas species (Sphingomonas yanoikuyae, Sphingomonas terrae and Sphingomonas macrogoltabidus) showed a second spot of SGL (SGL-1') identified as galacturonosyl ceramide. The presence of SGL-1 in cellular lipids suggested that strain $\mathrm{RW1}^{\top}$ is a member of the genus Sphingomonas. DNA-DNA reassociation rates between strain $\mathrm{RW1}^{\top}$ and each type strain of 14 Sphingomonas species including Sphingomonas paucimobilis, type species for the genus, revealed that strain RW1 $^{\top}$ is independent from these species. Results of phylogenetic analysis of $16 S$ rDNA sequences of strain RW1 ${ }^{\top}$ and type strains of 21 named Sphingomonas species verified that strain $\mathrm{RW1}^{\mathrm{T}}$ belongs to the genus Sphingomonas. Strain RW1 ${ }^{\top}$ could be differentiated from named species of the genus by phenotypic characteristics and has been assigned to a new species, Sphingomonas wittichii sp. nov. The type strain is DSM 6014 ${ }^{\top}\left(=\mathrm{JCM} 10273^{\top}=\right.$ EY 4224'). DNA G+C content is $67 \mathrm{~mol} \%$.
\end{abstract}

Keywords: dibenzo- $p$-dioxin metabolizer, Sphingomonas sp. strain $\mathrm{RW}^{\mathrm{T}}$, Sphingomonas wittichii sp. nov.

\section{INTRODUCTION}

From enrichment cultures inoculated with a water sample of the River Elbe, Wittich et al. (1992) isolated a strain that was able to grow with either dibenzo- $p$ dioxin (DD) or dibenzofuran (DF) as a sole source of carbon and energy; the strain was named Sphingomonas sp. strain $\mathrm{RW} 1^{\mathrm{T}}$. In addition to mineralization of $\mathrm{DD}$ and $\mathrm{DF}$, strain $\mathrm{RW} 1^{\mathrm{T}}$ co-metabolized monoand dichloro-derivatives of these compounds (Megharaj et al., 1997; Wilkes et al., 1996). The

Abbreviations: DD, dibenzo-p-dioxin; DF, dibenzofuran; SGL, sphingoglycolipid. enzymology of dioxygenation by strain $\mathrm{RW} 1^{\mathrm{T}}$ and its genetic study have been reported previously (Armengaud et al., 1998; Bünz et al., 1993; Bünz \& Cook, 1993).

$\mathrm{DD}, \mathrm{DF}$ and other related compounds are now known to be metabolized by certain species or strains of aerobic and anaerobic bacteria and fungi. Biotechnological methods can be used to exploit the degrading potential of such micro-organisms, which may be useful for the bioremediation of contaminated soil and water.

For the biodegradation of dioxin and related compounds, bacteriologists, mycologists, biochemists and geneticists are doing extensive work. Taxonomists 
have to support this research by polyphasic taxonomic techniques to establish nomenclatural standing of the organisms concerned.

The presence of the second spot of sphingoglycolipid (SGL), SGL-1', on TLC of a mild alkaline hydrolysate of cellular extractable lipids is a characteristic finding of $S$. yanoikuyae strains. This alkali-stable lipid was identified as galacturonosyl ceramide (Naka et al., 2000), which is also found in type strains of Sphingomonas wittichii sp. nov., Sphingomonas terrae and Sphingomonas macrogoltabidus.

\section{METHODS}

Bacterial strains. Since TLC of cellular lipids of strain $\mathrm{RW} 1^{\mathrm{T}}$ $\left(=\mathrm{DSM} 6014^{\mathrm{T}}=\mathrm{EY} 4224^{\mathrm{T}}\right)$, type strains of $S$. terrae EY $4207^{\mathrm{T}}$ and $S$. macrogoltabidus EY $4304^{\mathrm{T}}$ revealed a spot of SGL-1' (galacturonosyl ceramide) similar to that of Sphingomonas yanoikuyae EY $4208^{\mathrm{T}}$, these four strains were used, together with $S$. paucimobilis EY $2395^{\mathrm{T}}$, for comparative study of phenotypic characteristics. Strains were grown on either Bacto-heart infusion agar or half-concentration nutrient broth supplemented with $1.5 \%$ agar; incubations were at $30^{\circ} \mathrm{C}$, unless otherwise stated.

For DNA-DNA hybridization, in addition to the above described five strains, the type strains of ten other Sphingomonas species (Sphingomonas sanguinis, Sphingomonas parapaucimobilis, Sphingomonas adhaesiva, Sphingomonas capsulata, Sphingomonas trueperi, Sphingomonas chlorophenolica, Sphingomonas natatoria, Sphingomonas rosa, Sphingomonas echinoides and Sphingomonas mali), and Escherichia coli SMUM $344^{\mathrm{T}}$ as a control strain, were used (Table 1).
Morphological, biochemical and physiological characterization. For strain $\mathrm{RW} 1^{\mathrm{T}}$, motility was tested by microscopy of a wet mount preparation of young broth culture. Motile organisms were taken from diffuse growth on a $0.3 \%$ agar plate, inoculated with heart infusion broth; flagellation was confirmed by photomicroscopy of a flagella-stained preparation by Leifson's method. Transmission electron microscopy of cells negatively stained with $1 \%$ potassium phosphotungstate was performed under a JEOL JEM-1200 electron microscope.

Tests for characterization listed in Table 2 were performed by previously described methods (Yabuuchi et al., 1990). Utilization of citrate and malonate were determined by growth and alkali production on Simmons' citrate agar and in malonate broth (Eiken Chemicals), respectively. Oxidative acid production was tested by using Bacto-OF basal medium supplemented with a total of 26 carbohydrates and sugar alcohols $(1 \%)$ and ethanol $(3 \%)$. Results were read for up to 4 weeks. Biotype 100 and Biotype medium 1 or 2 (bioMérieux) were used to establish nutritional profiles of test organisms following the manufacturer's instructions.

Drug susceptibilities. Susceptibilities of the organisms were tested with 36 kinds of drug-containing Senci-discs and ready-to-use Mueller-Hinton medium II (BectonDickinson) as described previously (Yabuuchi et al., 1999).

Cellular lipids and fatty acids analysis. Cells grown on agar media were harvested, the cellular lipids were extracted twice, washed and hydrolysed with $0.5 \mathrm{M} \mathrm{NaOH}$ as described previously (Yabuuchi et al., 1990, 1999). The total extractable lipids and their alkaline hydrolysates were analysed by TLC with an acidic solvent system composed of chloroform/methanol/acetic acid/water (100:20:12:5, by vol.). Purified SGL-1 of S. paucimobilis

Table 1 Histories and corresponding numbers of the type strains of 15 Sphingomonas species and Escherichia coli

For comparative characterization, species nos 1-5 were used.

\begin{tabular}{|c|c|c|c|c|c|c|c|c|c|c|c|}
\hline \multirow[t]{2}{*}{ No. } & \multirow[t]{2}{*}{ Species } & \multicolumn{8}{|c|}{ Corresponding no.* } & \multirow[t]{2}{*}{ Received from:* } & \multirow[t]{2}{*}{ Received as: } \\
\hline & & ATCC & DSM & GIFU & IFO & JCM & NCTC & SMUM & $\mathbf{E Y}$ & & \\
\hline 1 & Sphingomonas sp. $\mathrm{RW} 1^{\mathrm{T}}$ & & 6014 & & & 10273 & & 2128 & 4224 & DSM & Sphingomonas sp. $\mathrm{RW} 1^{\mathrm{T}}$ \\
\hline 2 & S. yanoikuyae & 51230 & 7462 & 9882 & 15102 & 7371 & & 2121 & 4208 & Bruun & Sphingobacterium sp. AB 1105 \\
\hline 3 & S. terrae & 51381 & & & 15098 & 10195 & & 2120 & 4207 & $\mathrm{JCM}$ & Sphingomonas terrae \\
\hline 4 & S. macrogoltabidus & 51380 & & & 15033 & 10192 & & 3667 & 4304 & $\mathrm{JCM}$ & Sphingomonas macrogoltabidus \\
\hline 5 & S. paucimobilis $\dagger$ & 29837 & 1098 & 2395 & 13935 & 7516 & 11030 & 2114 & 2395 & NCTC & Pseudomonas paucimobilis \\
\hline 6 & S. sanguinis & 51382 & & 2397 & 13937 & 7514 & 11032 & 2115 & 2397 & GIFU & Sphingomonas genospecies 1 \\
\hline 7 & S. parapaucimobilis & 51231 & & 11387 & 15100 & 7510 & & 2122 & 4213 & GIFU & Sphingomonas parapaucimobilis \\
\hline 8 & S. adhaesiva & 51229 & & 11458 & 15099 & 7370 & & 2123 & 4215 & GIFU & Sphingomonas adhaesiva \\
\hline 9 & S. capsulata & 14666 & 30196 & 11526 & 12533 & 7508 & & 2124 & 4216 & GIFU & Sphingomonas capsulata \\
\hline 10 & S. trueperi & 12417 & & & & 10278 & & 2125 & 4218 & ATCC & Sphingomonas trueperi \\
\hline 11 & S. chlorophenolica & 33790 & & & & 10275 & & 2126 & 4219 & ATCC & Sphingomonas chlorophenolica \\
\hline 12 & S. natatoria & 35951 & 3183 & & & 10396 & & 2127 & 4220 & ATCC & Blastomonas natatoria \\
\hline 13 & S. rosa & 51837 & & & 15208 & 10277 & & 2131 & 4227 & $\mathrm{JCM}$ & Sphingomonas rosa \\
\hline 14 & S. echinoides & 14820 & 50409 & & 15742 & 10637 & & 3668 & 4340 & $\mathrm{JCM}$ & Sphingomonas echinoides \\
\hline \multirow[t]{2}{*}{15} & S. mali & 51840 & & & 15500 & 10193 & & 3669 & 4341 & IFO & Sphingomonas mali \\
\hline & Escherichia coli & 11775 & 30083 & 12542 & & 1649 & 9001 & 344 & 4342 & GIFU & Escherichia coli \\
\hline
\end{tabular}

* ATCC, American Type Culture Collection, Manassas, VA, USA; DSM, Deutsche Sammlung von Mikroorganismen und Zellkulturen GmbH, Braunschweig, Germany; GIFU, Department of Microbiology, Gifu University School of Medicine, Gifu, Japan; IFO, Institute of Fermentation Osaka, Osaka, Japan; JCM, Japan Collection of Microorganisms, Institute of Physical and Chemical Research (RIKEN), Saitama, Japan; NCTC, National Collection of Type Cultures, London, UK; SMUM, St Marianna University School of Medicine, Kanagawa, Japan; Bruun, E. Bruun, Copenhagen, Denmark; EY, Eiko Yabuuchi, Aichi Medical University, Aichi, Japan.

$\dagger$ Nomenclatural type species for the genus Sphingomonas. 
Table 2 Comparative characterization of Sphingomonas sp. RW1 ${ }^{\top}$ and four other Sphingomonas species

All five strains were Gram-negative, rod-shaped and positive for motility, growth at $30^{\circ} \mathrm{C}$, oxidase, catalase and hydrolysis of aesculin. All five strains were negative for growth on MacConkey agar, fermentation of glucose, nitrate to nitrite, acylamidase, phenylalanine deaminase, lysine, ornithine decarboxylase and arginine dihydrolase in decarboxylase base Møller, urease in Christensen's medium. ND, Not done.

\begin{tabular}{|c|c|c|c|c|c|}
\hline Characteristic & $\begin{array}{l}\text { Sphingomonas sp. strain } \\
\text { RW1 }^{\mathrm{T}}\left(=\mathrm{EY} 4224^{\mathrm{T}}\right)\end{array}$ & $\begin{array}{l}\text { S. yanoikuyae } \\
\text { EY } 4208^{\mathrm{T}}\end{array}$ & $\begin{array}{l}\text { S. terrae } \\
\text { EY } 4207^{\mathrm{T}}\end{array}$ & $\begin{array}{l}\text { S. macrogoltabidus } \\
\text { EY 4304 }\end{array}$ & $\begin{array}{l}\text { S. paucimobilis } \\
\text { EY } 23955^{\mathrm{T}}\end{array}$ \\
\hline Flagellation & Single polar/subpolar & Single polar & ND & ND & Single polar \\
\hline Deep yellow pigmentation & - & - & - & - & + \\
\hline \multicolumn{6}{|l|}{ Utilization of: } \\
\hline Citrate (Simmons) & - & + & + & + & + \\
\hline Malonate broth & + & - & + & - & - \\
\hline \multicolumn{6}{|l|}{ Hydrolysis of: } \\
\hline Gelatin & + & - & + & - & + \\
\hline Starch & - & - & - & & + \\
\hline Tween 80 & - & - & - & + & + \\
\hline Deoxyribonuclease & - & + & + & + & + \\
\hline $\mathrm{G}+\mathrm{C}(\mathrm{mol} \%)$ & $67 \dagger$ & $61 \cdot 7$ & $63 \cdot 0 \%$ & $63 \cdot 9$ & $63 \cdot 7$ \\
\hline
\end{tabular}

* Type species for the genus.

$\dagger$ Wittich et al. (1992).

† Takeuchi et al. (1993).

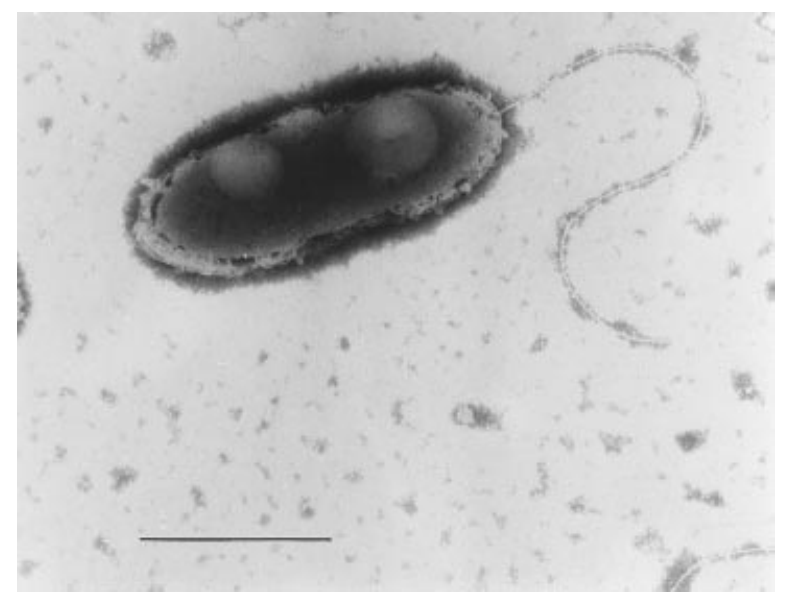

Fig. 1. Electron micrograph of a negatively stained cell of Sphingomonas sp. strain $\mathrm{RW}^{\top}$ with a single polar flagellum. Cells were stained with $1 \%$ potassium phosphotungstate and observed under a JEOL JEM-1200 transmission electron microscope. Bar, $0.5 \mu \mathrm{m}$

EY $2395^{\mathrm{T}}$ was used as a standard. To detect spots and their colour reaction, $50 \%$ sulfuric acid solution, Dittmer's reagent, ninhydrin solution and Anthrone reagent were used for all lipids, phospholipids, free amino bases and glycolipids, respectively. Cellular fatty acid analysis was performed by a method described previously (Yabuuchi et al., 1990, 1999).

Preparation of DNA. Bacterial cells growing in nutrient broth (Difco) in shaking culture for $24 \mathrm{~h}$ at $30^{\circ} \mathrm{C}$ were collected and resuspended in $50 \mathrm{ml} \mathrm{S}$-TE buffer $[10 \mathrm{mM}$ Tris $/ \mathrm{HCl}$ buffer, $1 \mathrm{mM}$ EDTA (disodium), $0 \cdot 15 \mathrm{M} \mathrm{NaCl}, \mathrm{pH} 8 \cdot 0$ ] containing lysozyme $\left(0 \cdot 1 \mathrm{mg} \mathrm{ml}^{-1}\right)$. The suspension, supplemented with $3 \% \mathrm{SDS}$, was incubated at $60^{\circ} \mathrm{C}$ for $10 \mathrm{~min}$. An equal volume of phenol mixture [phenol/chloroform/3-methyl-1-butanol (25:24:1, by vol.)] was added to the suspension, which was then mixed vigorously and centrifuged at $10000 \mathrm{~g}$ for $10 \mathrm{~min}$. The centrifuged supernatant fluid was transferred to a new tube and 2 vol. ethanol was added. The precipitated DNA dissolved in TE buffer $(10 \mathrm{mM}$ Tris $/ \mathrm{HCl}$ buffer, $1 \mathrm{mM}$ EDTA, pH 8.0) was incubated with RNase A $\left(10 \mu \mathrm{g} \mathrm{ml}^{-1}\right)$ for $30 \mathrm{~min}$ at $35^{\circ} \mathrm{C}$ and further incubated with proteinase $\mathrm{K}$ $\left(10 \mu \mathrm{g} \mathrm{ml}^{-1}\right)$ and $0 \cdot 3 \% \mathrm{SDS}$ for $60 \mathrm{~min}$ at $50{ }^{\circ} \mathrm{C}$. Following phenol mixture treatment and ethanol precipitation, the purified DNA was dissolved in TE buffer and used as a reference for DNA-DNA hybridization.

Preparation of DNA plate and labelled DNA. Immobilization of the reference DNA to a microplate was performed by a previously described protocol (Ezaki et al., 1989.). The heatdenatured reference DNA $\left(10 \mu \mathrm{g} \mathrm{ml}^{-1}\right)$ was dissolved in PBS $(0 \cdot 145 \mathrm{M} \mathrm{NaCl}, 0 \cdot 15 \mathrm{M}$ sodium phosphate) containing $0 \cdot 1 \mathrm{M}$ $\mathrm{MgCl}_{2}$. A portion $(100 \mu \mathrm{l})$ of the solution was distributed into wells of the microplate (Cliniplate EB; Labsystems). The plates were kept for $16 \mathrm{~h}$ at $30{ }^{\circ} \mathrm{C}$. After discarding the solution, the DNA plate was kept in a dry condition. The DNA was labelled using a commercial kit with a photoactivatable form of biotin (PHOTOPROBE Long Arm Biotin, SP-1020; Vector Laboratories).

Hybridization. Blocking solution $[2 \times \mathrm{SSC}(0 \cdot 3 \mathrm{M} \mathrm{NaCl}$ and $0.03 \mathrm{M}$ sodium citrate) containing $2 \%$ blocking reagent (Boehringer Mannheim)] was distributed to wells of the DNA plate and kept for $30 \mathrm{~min}$ at room temperature. After discarding the blocking solution, $100 \mu \mathrm{l}$ hybridization solution $(2 \times \mathrm{SSC}, 50 \%$ formamide, $1 \%$ blocking reagent; pH 7.2-7.6) containing labelled single-stranded DNA was distributed to wells of the DNA plate. After $16 \mathrm{~h}$ hybridization at $50{ }^{\circ} \mathrm{C}$, the wells were washed three times 


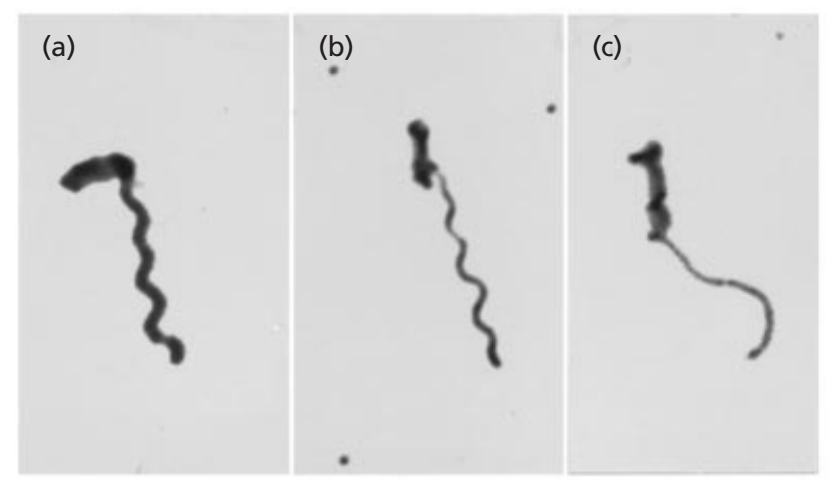

Fig. 2. Flagellar morphology of Sphingomonas sp. strain $R W 1^{\top}$ stained by Leifson's method. Three images ( $a, b$ and $c)$ were taken from the same smear. (a) A single polar flagellum of normal type wave; (b) a single subpolar flagellum of normal type wave; (c) a single polar flagellum of a single long curvature.

with $1 \times$ SSC solution. Alkaline phosphatase-streptoavidin (Vector Laboratories) was diluted 1000-fold with PBS containing blocking agent (Block Ace Powder; Dainippon
Seiyaku). The diluted mixture was distributed to the wells $\left(100 \mu \mathrm{l}\right.$ in each). After $30 \mathrm{~min}$ incubation at $35^{\circ} \mathrm{C}$, the wells were washed three times with PBS and filled with $100 \mu \mathrm{l}$ substrate solution (BluePho Microwell Phosphatase Substrate system; Kirkegaard \& Perry Laboratories). The plate was incubated at room temperature and the $\mathrm{OD}_{630}$ of each well was measured. The relative relatedness among the reference DNAs was calculated using the $\mathrm{OD}_{630}$ values and the protocol of colorimetric microplate hybridization (Kusunoki et al., 1991).

16S rDNA gene sequences and phylogenetic analysis. Deposited data on $16 \mathrm{~S}$ rDNA sequences obtained from the DNA Database of Japan (DDBJ, Institute of Genetics, Mishima, Shizuoka, Japan) were used for the phylogenetic analysis. In addition to the five strains listed in Table 1, data of type strains of 17 Sphingomonas species and two subspecies of Zymomonas mobilis were used. Brevundimonas diminuta DSM $1635^{\mathrm{T}}$ was used as an outgroup organism. Sources of sequence data of a total of 25 strains are shown in parentheses following each type strain number in the phylogenetic tree (Fig. 4).

For the phylogenetic analysis, alignment gaps and unidentified base positions were not taken into consideration for the calculation. Each 1210 base sequence of all 25 strains (Fig. 4) was used for the calculation. Nucleotide substitution rates $\left(K_{\text {nuc }}\right)$ (Kimura, 1980) were determined by the CLUSTAL

Table 3 Oxidative acid production from 26 carbohydrates, sugar alcohols and ethanol by Sphingomonas sp. strain $\mathrm{RWI}^{\top}$ and type strains of five other Sphingomonas spp.

The first six rows of the table show tests useful for differentiation of strain $\mathrm{RW} 1^{\mathrm{T}}$. + , Positive reaction within $3 \mathrm{~d}$; $+{ }^{*}, 4 \mathrm{~d}$ or more required for positive reaction; -, negative reaction during 4 week incubation; NG, no growth.

\begin{tabular}{|c|c|c|c|c|c|c|}
\hline Substrate & $\begin{array}{l}\text { Strain RW1 } \\
(=\text { EY 4224 } \\
(=2\end{array}$ & $\begin{array}{l}\text { S. yanoikuyae } \\
\text { EY } 4208^{\mathrm{T}}\end{array}$ & $\begin{array}{c}\text { S. mali } \\
\text { EY } 4340^{\mathrm{T}}\end{array}$ & $\begin{array}{l}\text { S. terrae } \\
\text { EY } 4207^{\mathrm{T}}\end{array}$ & $\begin{array}{l}\text { S. macrogoltabidus } \\
\text { EY } 4304^{\mathrm{T}}\end{array}$ & $\begin{array}{l}\text { S. paucimobilis } \\
\text { EY } 2395^{\mathrm{T}} \dagger\end{array}$ \\
\hline Inulin & $+^{*}$ & - & - & - & + & - \\
\hline Sorbitol & + & - & - & - & - & - \\
\hline Mannitol, D-ribose & + & - & + & + & - & - \\
\hline Fructose, galactose & + & + & - & - & - & + \\
\hline Dulcitol & - & - & - & + & - & $+*$ \\
\hline Xylose & - & + & - & - & - & + \\
\hline Glycerol & + & + & - & - & - & $+^{*}$ \\
\hline Maltose & + & + & + & + & - & $+*$ \\
\hline Glucose & + & + & - & $+*$ & - & $+*$ \\
\hline Salicin & + & + & - & + & - & $+*$ \\
\hline Rhamnose & + & + & + & + & - & - \\
\hline D-Ribose & + & - & $+^{*}$ & + & - & - \\
\hline Cellobiose & $+^{*}$ & + & $+^{*}$ & + & + & $+*$ \\
\hline Sucrose & $+*$ & + & $+*$ & $+*$ & + & $+*$ \\
\hline Mannose, melezitose & $+*$ & + & $+^{*}$ & + & - & + \\
\hline Trehalose & $+*$ & + & + & + & + & $+*$ \\
\hline D-Arabinose & - & + & + & - & - & $+*$ \\
\hline L-Arabinose & - & + & $+^{*}$ & - & + & $+*$ \\
\hline Melibiose & - & + & + & $+^{*}$ & + & - \\
\hline Ethanol & - & + & - & $+^{*}$ & NG & + \\
\hline Raffinose & - & + & - & $+*$ & - & $+*$ \\
\hline Inositol & - & - & - & $+*$ & - & - \\
\hline Adonitol & - & - & - & - & - & - \\
\hline Lactose & - & + & - & - & - & $+^{*}$ \\
\hline
\end{tabular}

$\dagger$ Type species of the genus. 
w program (Thompson et al., 1994). The neighbour-joining method (Saitou \& Nei, 1987) was used to reconstruct a phylogenetic tree from the distance matrices by NJPLOT written by Manolo Gouy (Laboratoire de Biometrie, University of Lyon, Villeurbanne, France). To evaluate the topology of the phylogenetic tree, a bootstrap analysis was performed. Bootstrap values, out of a total of 1000 replications, are indicated at the branching points.

\section{RESULTS}

\section{Phenotypic characteristics}

Colonies of strain $\mathrm{RW}^{\mathrm{T}}\left(=\mathrm{EY} 4224^{\mathrm{T}}\right)$ were $1 \mathrm{~mm}$ in diameter, smooth and glistening with entire margins and faintly yellow-pigmented after $3 \mathrm{~d}$ incubation at $30{ }^{\circ} \mathrm{C}$. Compared with $S$. yanoikuyae EY $4208^{\mathrm{T}}$, S. macrogoltabidus EY $4304^{\mathrm{T}}$ and S. paucimobilis $\mathrm{EY}$
$2395^{\mathrm{T}}$, growth of strain RW1 $1^{\mathrm{T}}$ and $S$. terrae EY $4207^{\mathrm{T}}$ was slower; $3 \mathrm{~d}$ were required for pin-point colony production. Cells of strain $\mathrm{RW} 1^{\mathrm{T}}$ were Gram-negative straight rods with rounded ends. Soma size was $0.4-0.6 \times 0.9-1.5 \mu \mathrm{m}$. Cells were actively motile with a single polar or subpolar flagellum (Figs 1 and 2).

A comparison of phenotypic characteristics of type strains of five Sphingomonas species is given in Table 2 . Strain $R W 1^{\mathrm{T}}$ produced acid oxidatively from 18 of 26 carbohydrates, sugar alcohols and ethanol in OF basal medium (Difco). Positive reactions were observed for 12 carbohydrates, including glucose and inulin, within $3 \mathrm{~d}$ of inoculation. Acid was slowly produced from the remaining eight carbohydrates. Oxidative acid production in OF medium is summarized in Table 3.

Results of carbon assimilation using Biotype 100 (99

Table 4 Assimilation test (Biotype 100) results for Sphingomonas sp. strain $\mathrm{RW}^{\top}{ }^{\top}$ and the type strains of four Sphingomonas species

The names of the four Sphingomonas species used are given in Table 2. All five strains were positive for: aesculin, DL- $\beta$ hydroxybutyrate, L-glutamate, $\mathrm{D}(+)$ trehalose, maltotriose and maltose. All five strains were negative for: $\mathrm{D}(+)$ arabitol, L( - )arabitol, xylitol, dulcitol, D-sorbitol, adonitol, malonate, D-lyxose, $i$-erythritol, hydroxyquinoline- $\beta$-glucuronate, $\mathrm{L}(+)$ tartrate, tryptamine, itaconate, $\mathrm{L}(+)$ sorbose, $\alpha$-ketoglutarate, meso-tartrate, $\mathrm{D}(+)$ malate, D-galacturonate, 2-keto-D-gluconate, 5ketogluconate, L-tryptophan, $m$-hydroxybenzoate, benzoate, 3-phenylpropionate, $m$-coumarate, trigonellin, putrescine, histamine, caprylate, L-histidine, DL- $\alpha$-amino-n-valerate, ethanolamine and $\alpha$-ketogluconate.

\begin{tabular}{|c|c|c|c|}
\hline \multirow[t]{2}{*}{ Substrate } & \multirow{2}{*}{$\begin{array}{c}\text { Result of strain } \\
\operatorname{RW1}^{\mathrm{T}}\left(=\mathrm{EY} \mathrm{4224}{ }^{\mathrm{T}}\right)\end{array}$} & \multicolumn{2}{|c|}{ EY no. of type strain giving the result: } \\
\hline & & + & - \\
\hline $\begin{array}{l}\text { D-Tagatose, myo-inositol, tricarballylate, } \\
\text { phenylacetate, DL-glycerate, D-glucosamine }\end{array}$ & + & & $4208,4207,4304,2395$ \\
\hline $\begin{array}{l}\alpha \text {-D }(+) \text { Glucose, } \beta \text {-D }(+) \text { fructose, } \mathrm{D}(+) \text { xylose, } \\
\alpha \text {-D }(+) \text { melibiose, } \mathrm{D}(+) \text { raffinose, } \mathrm{D}(+) \text { galactose, } \\
\alpha \text {-lactose, } \mathrm{D}(+) \text { cellobiose, } \beta \text {-gentiobiose, DL-lactate, } \\
\text { L-aspartate, } \mathrm{L}(+) \text { arabinose, sucrose, } \mathrm{D}(+) \text { turanose, } \\
N \text {-acetyl-D-glucosamine, maltitol, } \\
\text { 1-o-methyl- } \beta \text {-glucopyranoside }\end{array}$ & + & $4208,4304,2395$ & 4207 \\
\hline L-Proline & + & $4208,4207,4304$ & 2395 \\
\hline L-Serine, L-alanine & + & $4207,4304,2395$ & 4208 \\
\hline $\mathrm{L}(-)$ Malate, succinate, fumarate & + & $4208,4207,2395$ & 4304 \\
\hline L-Tyrosine & + & 4304,2395 & 4208,4207 \\
\hline $\begin{array}{l}\text { 1-o-Methyl- } \alpha \text {-D-glucopyranoside } \\
\mathrm{D}(+) \text { Mannose, }(-) \text { quinate }\end{array}$ & + & 4208,2395 & 4207,4304 \\
\hline Glycerol, D-gluconate & + & 4208,4304 & 4207,2395 \\
\hline Citrate, D-glucuronate, gentisate & + & 4208 & $4207,4304,2395$ \\
\hline D-Alanine & + & 4207 & $4208,4304,2395$ \\
\hline D-Mannitol, DL- $\alpha$-amino-n-butyrate & + & 4304 & $4208,4207,4304$ \\
\hline cis-Aconitate, trans-aconitate & + & 2395 & $4208,4207,4304$ \\
\hline Propionate & - & $4208,4207,4304$ & 2395 \\
\hline Paratinose, protocatechuate, & - & $4208,4304,2395$ & 4207 \\
\hline $\mathrm{L}(-)$ Fucose, $\mathrm{D}(+)$ melezitose, $p$-hydroxybenzoate & - & 4208,2395 & 4207,4304 \\
\hline D-Gluconate, glycerol & - & 4208,4304 & 4207,2395 \\
\hline$\alpha$-L-Rhamnose, D-saccharate, caprate, mucate & - & 4208 & $4207,4304,2395$ \\
\hline $\mathrm{D}(-)$ Tartrate & - & 4207 & $4208,4304,2395$ \\
\hline D-Ribose & - & 4304 & $4208,4207,2395$ \\
\hline Betaine, glutarate & - & 2395 & $4208,4207,4304$ \\
\hline
\end{tabular}


Table 5 Susceptibilities of Sphingomonas sp. strain $\mathrm{RW} 1^{\top}$ and four other Sphingomonas species against 36 antibacterial agents by the agar disk diffusion method

S, Susceptible; R, resistant; IM, intermediate.

\begin{tabular}{|c|c|c|c|c|c|c|}
\hline Drug & $\begin{array}{l}\text { Strain } \\
\text { RW1 }\end{array}$ & $\begin{array}{l}\text { S. yanoikuyae } \\
\text { EY } 4208^{\mathrm{T}}\end{array}$ & $\begin{array}{l}\text { S. terrae } \\
\text { EY } 4207^{\mathrm{T}}\end{array}$ & $\begin{array}{l}\text { S. macrogoltabidus } \\
\text { EY } 4304^{\mathrm{T}}\end{array}$ & $\begin{array}{l}\text { S. paucimobilis } \\
{\text { EY } 2395^{\mathrm{T}}}^{2}\end{array}$ & $\begin{array}{l}\text { No. resistant } \\
\text { strains }\end{array}$ \\
\hline $\begin{array}{l}\text { Imipenem, tetracycline, doxycycline, } \\
\text { minocycline }\end{array}$ & $\mathrm{S}$ & $\mathrm{S}$ & $\mathrm{S}$ & $\mathrm{S}$ & $\mathrm{S}$ & 0 \\
\hline $\begin{array}{l}\text { Amikacin, gentamicin, dibekacin, } \\
\text { panipenem }\end{array}$ & $\mathrm{S}$ & $\mathrm{S}$ & $\mathrm{S}$ & $\mathrm{S}$ & $\mathrm{S}$ & 0 \\
\hline Amoxicillin/clavulanic acid & $\mathrm{S}$ & $\mathrm{S}$ & $\mathrm{S}$ & IM & $\mathrm{S}$ & 0 \\
\hline $\begin{array}{l}\text { Cefaclor, ceftazidime, cefotaxim, } \\
\text { cefoperazon }\end{array}$ & $\mathrm{S}$ & $\mathrm{S}$ & IM & $\mathrm{R}$ & $\mathrm{S}$ & 1 \\
\hline $\begin{array}{l}\text { Penicillin, ampicillin, amoxicillin, } \\
\text { meropenem }\end{array}$ & $\mathrm{S}$ & $\mathrm{S}$ & $S$ & $\mathrm{R}$ & $\mathrm{S}$ & 1 \\
\hline $\begin{array}{l}\text { Sparfloxacin, levofloxacin, ciprofloxacin, } \\
\text { ofloxacin }\end{array}$ & $\mathrm{R}$ & $\mathrm{S}$ & $\mathrm{S}$ & $\mathrm{S}$ & $\mathrm{S}$ & 1 \\
\hline Tosufloxacin, norfloxacin, erythromycin & $\mathrm{R}$ & IM & IM & $\mathrm{S}$ & $\mathrm{S}$ & 1 \\
\hline Polymyxin B & IM & $\mathrm{S}$ & $\mathrm{R}$ & $\mathrm{S}$ & IM & 1 \\
\hline $\begin{array}{l}\text { Clarithromycin, sulfamethoxazole/ } \\
\text { trimethoprim }\end{array}$ & $\mathrm{R}$ & $\mathrm{S}$ & $\mathrm{R}$ & $\mathrm{S}$ & $\mathrm{S}$ & 2 \\
\hline Roxithromycin & $\mathrm{R}$ & $\mathrm{R}$ & $\mathrm{R}$ & $\mathrm{IM}$ & $\mathrm{S}$ & 3 \\
\hline Flomoxef, carmonum & $\mathrm{S}$ & $\mathrm{R}$ & $\mathrm{R}$ & $\mathrm{R}$ & $\mathrm{S}$ & 3 \\
\hline Piperacillin, cefazolin & $\mathrm{S}$ & $\mathrm{R}$ & $\mathrm{S}$ & $\mathrm{R}$ & $\mathrm{R}$ & 3 \\
\hline Cefmetazole & $\mathrm{S}$ & $\mathrm{R}$ & $\mathrm{R}$ & $\mathrm{R}$ & $\mathrm{R}$ & 4 \\
\hline Aztreonam & $\mathrm{R}$ & $\mathrm{R}$ & $\mathrm{R}$ & $\mathrm{R}$ & $\mathrm{IM}$ & 4 \\
\hline Moxalactam, trimethoprim & $\mathrm{R}$ & $\mathrm{R}$ & $\mathrm{R}$ & $\mathrm{R}$ & $\mathrm{R}$ & 5 \\
\hline \multicolumn{7}{|l|}{ Total number of drugs } \\
\hline Resistant & 13 & 9 & 10 & 16 & 5 & \\
\hline Intermediate & 1 & 3 & 7 & 2 & 2 & \\
\hline Susceptible & 22 & 24 & 19 & 18 & 29 & \\
\hline Susceptible + Intermediate & 23 & 27 & 26 & 20 & 31 & \\
\hline
\end{tabular}

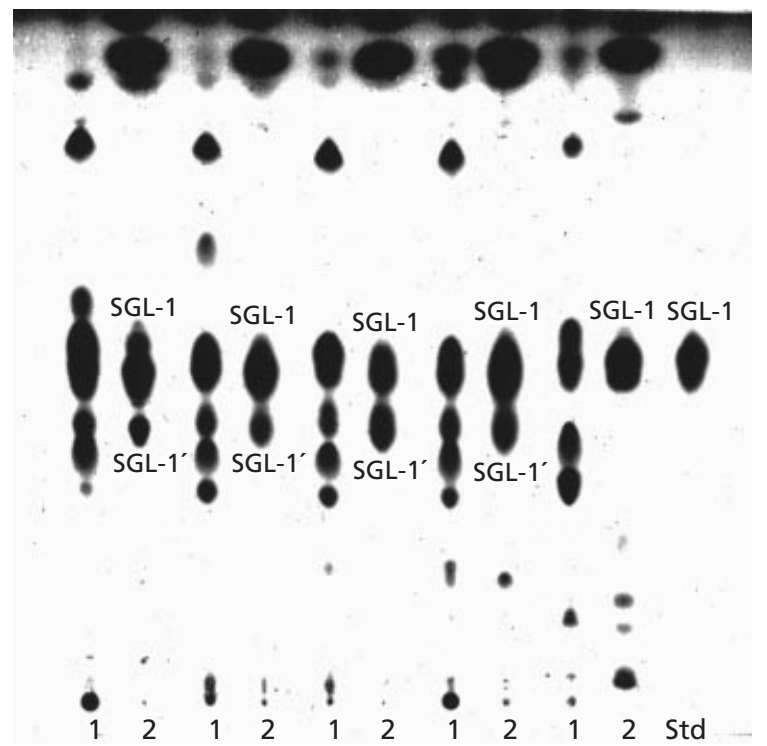

Fig. 3. TLC of cellular lipids of strain $\mathrm{RW} 1^{\top}\left(=\mathrm{EY} 4224^{\top}\right)$, S. yanoikuyae $\mathrm{EY} 4208^{\top}$, S. terrae $\mathrm{EY} 4207^{\top}$, S. macrogoltabidus EY $4304^{\top}$, S. paucimobilis EY $2395^{\top}$ and purified SGL-1 of EY $2395^{\top}$ as standard (from left to right). Solvent system: chloroform/methanol/acetic acid/water (100:20:12:5, by vol.). For each strain, lane 1 indicates a crude lipid and lane 2 indicates an alkali-stable lipid. 
Table 6 Cellular fatty acid composition of Sphingomonas sp. strain RW1 ${ }^{\top}$ and type strains of four Sphingomonas species

- , Not detected; TR, trace (less than $1 \%$ ).

\begin{tabular}{|c|c|c|c|c|c|}
\hline Fatty acid & $\begin{array}{l}\text { Strain RW1 } \\
(=\text { EY 4224 } \\
(=0\end{array}$ & $\begin{array}{l}\text { S. yanoikuyae } \\
\text { EY } 4208^{\mathrm{T}}\end{array}$ & $\begin{array}{l}\text { S. terrae } \\
\text { EY } 4207^{\mathrm{T}}\end{array}$ & $\begin{array}{c}\text { S. macrogoltabidus } \\
\text { EY } 4304^{\mathrm{T}}\end{array}$ & $\begin{array}{l}\text { S. paucimobilis } \\
\text { EY } 2395^{\mathrm{T} *}\end{array}$ \\
\hline $14: 0$ & TR & TR & - & TR & 1 \\
\hline $15: 0$ & - & - & 2 & TR & - \\
\hline cis9-16:1 & 6 & 14 & 4 & 29 & 2 \\
\hline $16: 1 \dagger$ & 2 & 2 & TR & 3 & - \\
\hline $16: 0$ & 12 & 9 & 2 & 10 & 17 \\
\hline $17: 1$ & - & & 8 & - & - \\
\hline $17: 0 \Delta$ & TR & TR & 45 & TR & - \\
\hline $17: 0$ & - & TR & 4 & - & - \\
\hline cis $11-$ and trans $9-18: 1$ & 54 & 52 & 13 & 43 & 60 \\
\hline $18: 1 \ddagger$ & TR & 2 & TR & TR & - \\
\hline $18: 0$ & - & TR & TR & TR & 1 \\
\hline $19: 1$ & - & - & TR & - & - \\
\hline $19: 0 \Delta$ & 3 & - & 1 & - & TR \\
\hline 2-OH 14:0 & 10 & 14 & 2 & 7 & 18 \\
\hline 2-OH 15:0 & - & - & 13 & 2 & - \\
\hline 2-OH 16:1 & - & $\mathrm{TR}$ & - & - & - \\
\hline 2-OH 16:0 & - & 2 & $\mathrm{TR}$ & $\mathrm{TR}$ & - \\
\hline Others & 11 & 3 & 2 & 3 & TR \\
\hline
\end{tabular}

* Type species of the genus.

$\dagger$ Except cis9-16:1.

† Except cis $11-$ and trans9-18:1.

Table 7 DNA-DNA reassociation rates (\%) among the type strains of 15 Sphingomonas species and Escherichia coli SMUM 344

SMUM, Department of Microbiology, St Marianna University School of Medicine, Kanagawa, Japan; EY, Eiko Yabuuchi, Department of Microbiology, Aichi Medical University, Aichi, Japan.

\begin{tabular}{|c|c|c|c|c|c|c|c|c|c|c|c|c|c|c|c|c|}
\hline \multirow[t]{2}{*}{ EY no. } & \multirow{2}{*}{ Species } & \multicolumn{15}{|c|}{ DNA from: } \\
\hline & & $4224^{T}$ & 4208 & 4207 & 4304 & 2395 & 2397 & 4213 & 4215 & 4216 & 4218 & 4219 & 4220 & 4227 & 4340 & 4341 \\
\hline $4224^{\mathrm{T}}$ & $\begin{array}{l}\text { Sphingomonas } \\
\text { sp. strain } \mathrm{RW} 1^{\mathrm{T}}\end{array}$ & 100 & 3 & 2 & 3 & 1 & 5 & 3 & 3 & 3 & 16 & 3 & 0 & 10 & 5 & 5 \\
\hline 4208 & S. yanoikuyae & 0 & 100 & 0 & 2 & 2 & 5 & 3 & 18 & 8 & 3 & 16 & 0 & 11 & 3 & 5 \\
\hline 4207 & S. terrae & 0 & 1 & 100 & 2 & 27 & 1 & 1 & 0 & 1 & 0 & 1 & 0 & 1 & 1 & 0 \\
\hline 4304 & S. macrogoltabidus & 0 & 2 & 22 & 100 & 1 & 1 & 1 & 0 & 2 & 0 & 3 & 3 & 3 & 1 & 1 \\
\hline 2395 & S. paucimobilis* & 0 & 4 & 2 & 1 & 100 & 33 & 24 & 1 & 10 & 9 & 4 & 0 & 6 & 4 & 6 \\
\hline 2397 & S. sanguinis & 0 & 7 & 4 & 0 & 29 & 100 & 35 & 1 & 3 & 4 & 5 & 3 & 7 & 4 & 5 \\
\hline 4213 & S. parapaucimobilis & 1 & 7 & 1 & 0 & 36 & 42 & 100 & 0 & 2 & 5 & 5 & 0 & 7 & 4 & 6 \\
\hline 4215 & S. adhaesiva & 6 & 12 & 0 & 0 & 11 & 13 & 7 & 100 & 6 & 5 & 14 & 0 & 6 & 8 & 5 \\
\hline 4216 & S. capsulata & 0 & 6 & 6 & 0 & 1 & 2 & 2 & 0 & 100 & 0 & 0 & 0 & 4 & 1 & 1 \\
\hline 4218 & S. trueperi & 1 & 4 & 6 & 0 & 5 & 5 & 2 & 0 & 3 & 100 & 6 & 0 & 2 & 2 & 2 \\
\hline 4219 & S. chlorophenolica & 0 & 8 & 5 & 0 & 1 & 1 & 1 & 0 & 3 & 0 & 100 & 0 & 6 & 2 & 1 \\
\hline 4220 & S. natatoria & 0 & 3 & 4 & 0 & 1 & 2 & 2 & 0 & 32 & 0 & 4 & 100 & 6 & 1 & 1 \\
\hline 4227 & S. rosa & 1 & 3 & 0 & 2 & 1 & 6 & 2 & 0 & 6 & 0 & 3 & 0 & 100 & 1 & 2 \\
\hline 4340 & S. echinoides & 0 & 3 & 8 & 2 & 4 & 7 & 2 & 0 & 3 & 3 & 8 & 8 & 3 & 100 & 4 \\
\hline 4341 & S. mali & 5 & 3 & 7 & 1 & 14 & 9 & 1 & 0 & 1 & 8 & 2 & 0 & 4 & 2 & 100 \\
\hline 4342 & Escherichia coli SMUM 344 & 0 & 0 & 0 & 0 & 0 & 0 & 0 & 0 & 0 & 0 & 0 & 0 & 0 & 0 & 0 \\
\hline
\end{tabular}

* Type species of the genus. 


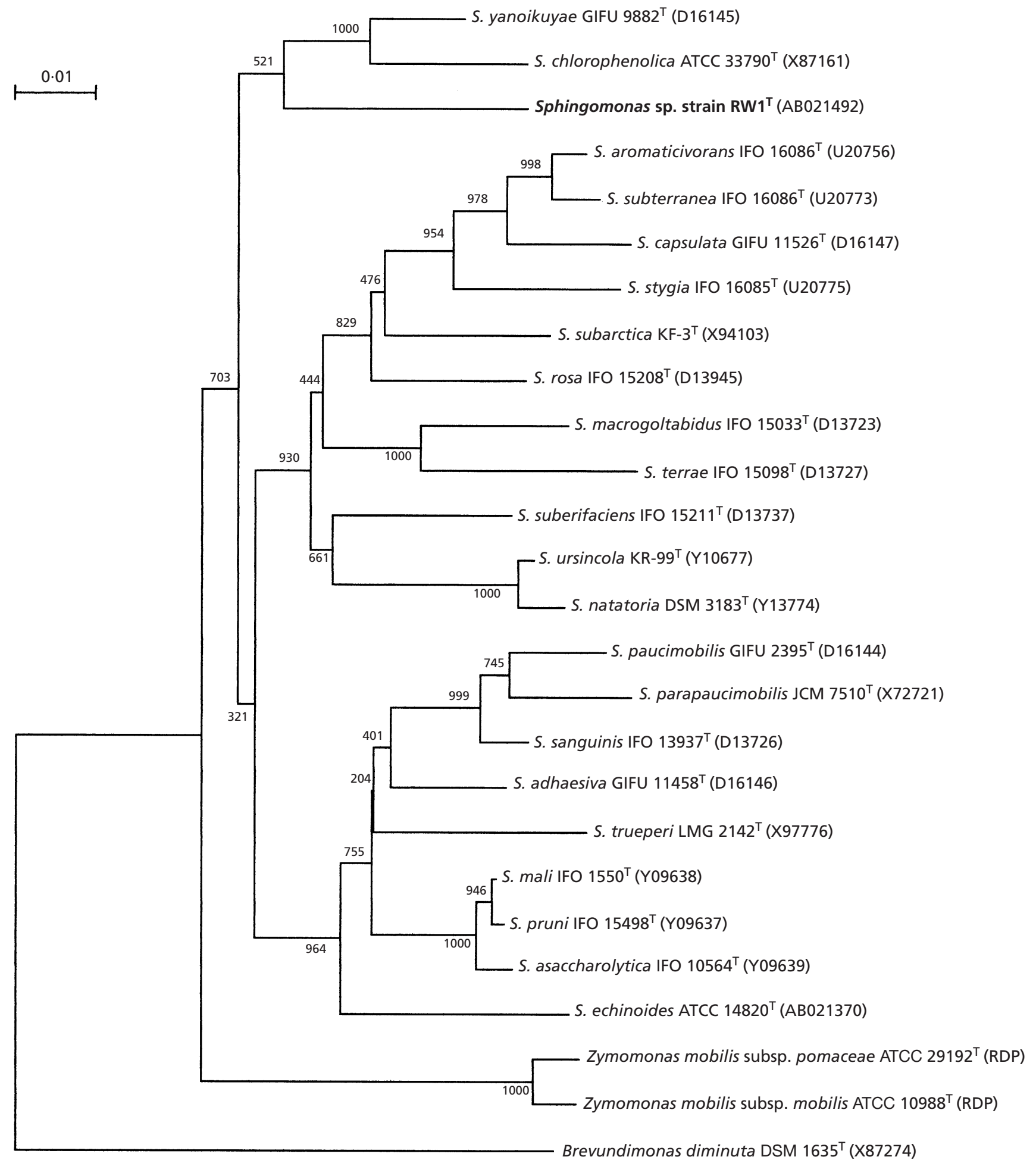

Fig. 4. Phylogenetic position of Sphingomonas sp. strain $\mathrm{RW} 1^{\top}$ among Sphingomonas and Zymomonas species obtained by the neighbour-joining analysis of $16 \mathrm{~S}$ rDNA. Figures in parentheses indicate the accession numbers of $16 \mathrm{~S}$ rDNA sequence data. Bar, 10 nucleotide substitutions per 1000 nucleotides of $16 \mathrm{~S}$ rDNA sequence. Bootstrap values from 1000 analyses are shown at the branch points.

substrates and negative control) are shown in Table 4. Whereas $S$. paucimobilis EY $2395^{\mathrm{T}}$ and $S$. yanoikuyae EY $4208^{\mathrm{T}}$ assimilated 44 and 47 substrates, respect- ively, strain RW1 $1^{\mathrm{T}}$ assimilated 50. S. terrae EY $4207^{\mathrm{T}}$ and $S$. macrogoltabidus EY $4304^{\mathrm{T}}$ assimilated 16 and 38 substrates, respectively. 
Strain $\mathrm{RW} 1^{\mathrm{T}}$ was susceptible to penicillins, cephems, macrolides and aminoglycosides, whereas it was characteristically resistant to all seven new quinolone chemotherapeutics tested (Table 5).

\section{Cellular lipids and fatty acids analysis}

Major and ubiquitous cellular lipids of all 15 strains tested were phosphatidyl ethanolamine, phosphatidyl glycerol and cardiolipin (data not shown). As shown in Fig. 3, which was developed by an acidic solvent system, spots of SGL-1' were seen in the alkali-stable lipids of RW1 ${ }^{\mathrm{T}}$, S. yanoikuyae $\mathrm{EY} 4208^{\mathrm{T}}$, S. terrae $\mathrm{EY}$ $4207^{\mathrm{T}}$ and S. macrogoltabidus EY $4304^{\mathrm{T}}$.

The major cellular fatty acid was $18: 1$ in four strains, with the exception of $S$. terrae EY $4207^{\mathrm{T}}$ in which 17:0 cyclopropanoic acid was the major fatty acid. The major 2-hydroxy acid in S. terrae EY $4207^{\mathrm{T}}$ was $2-\mathrm{OH}$ 15:0, whereas $2-\mathrm{OH} 14: 0$ was the major acid in the four other strains. None of the five strains contained 3hydroxy fatty acids (Table 6).

\section{DNA-DNA reassociation rates and phylogenetic analysis of $16 \mathrm{~S}$ rDNA sequences}

As shown Table 7, DNA-DNA reassociation rates between strain RW $1^{\mathrm{T}}$ and each type strain of the 14 Sphingomonas species, including three species $(S$. yanoikuyae, $S$. terrae and $S$. macrogoltabidus) which contain SGL-1' ${ }^{\prime}$ in their cellular lipids, ranged from 0 to $10 \%$. Reassociation rates among the 14 species ranged from 0 to $42 \%$ and also reconfirmed them as independent species.

The phylogenetic tree (Fig. 4) illustrates that strain $\mathrm{RW} 1^{\mathrm{T}}$ falls within the genus Sphingomonas and shows clear distinction from the 14 named species of this genus. The type strains of the four SGL-1'-containing species did not form a cluster.

\section{DISCUSSION}

Since the transfer of Pseudomonas paucimobilis (Holmes et al., 1977) to a new genus, Sphingomonas, biodegradation or utilization of DD, DF and other various related compounds by strains of Sphingomonas species have been reported. Examples of such compounds are diphenyl ether (Schmidt et al., 1992), 4,4'and 2,4-dihalodiphenyl ethers (Schmidt et al., 1993), DD (Wittich et al., 1992), chlorinated DF (Wilkes et al., 1996; Strubel et al., 1989), chloro- and bromobiphenyls (Davison et al., 1996), polycyclic aromatic hydrocarbons (Khan et al., 1996; Dagher et al., 1997), pentachlorophenol (Nohynek et al., 1995; Karlson et al., 1996; McCarthy et al., 1996), herbicides (Nickel et al., 1997), polyethylene glycol (Takeuchi et al., 1993; Zipper et al., 1996) and $\gamma$-hexachlorocyclohexane (Thomas et al., 1996).

Enzymes (Armengaud \& Timmis, 1997; Bünz et al., 1993; Bünz \& Cook, 1993; Happe et al., 1993; McCarthy et al., 1996), plasmids (Yrjala et al., 1997, 1998; Romine et al., 1999) and genetics (Armengaud et al., 1998; Masai et al., 1999; Miyauchi et al., 1998; Peng et al., 1998) of such degradations have also been reported.

Most of the organisms studied in the above-described literature are named as Sphingomonas sp., without establishing their nomenclatural standing as a species. Sphingomonas sp. strain $\mathrm{RW} 1^{\mathrm{T}}$ has been known as the most potent organism to degrade and even utilize DD and DF as sole sources of carbon and energy (Wittich et al., 1992). DNA-DNA reassociation rates between the type strains of each of 14 Sphingomonas species studied revealed that strain $\mathrm{RW} 1^{\mathrm{T}}$ is independent from these species. The presence of SGL-1 (Fig. 3) and 2$\mathrm{OH}$ myristic acid, and the absence of 3-OH fatty acids (Table 6) in cellular lipids suggests that $\mathrm{RW}^{\mathrm{T}}$ is a member of the genus Sphingomonas; this is confirmed by phylogenetic analysis of the $16 \mathrm{~S}$ rDNA sequence (Fig. 4). Among the 22 Sphingomonas species, only $S$. terrae, S. macrogoltabidus, S. yanoikuyae and strain RW ${ }^{\mathrm{T}}$ contained SGL-1' (galacturonosyl ceramide) in their cellular lipids (Fig. 3). These four organisms did not form a cluster in $16 \mathrm{~S}$ rDNA phylogenetic analysis (Fig. 4) and could be phenotypically differentiated from each other (Table 8). Although it has been previously reported (Naka et al., 2000) that $S$. mali EY 4206 possesses SGL-1' as its cellular lipid component, the strain was identified as $S$. macrogoltabidus by DNA-DNA hybridization. In addition, cellular lipids of $S$. mali EY $4341^{\mathrm{T}}$, obtained from IFO as IFO $15500^{\mathrm{T}}$, did not contain SGL-1'. These findings indicate that S. mali EY 4206 used in the previous study (Naka et al., 2000) was, in fact, a strain of $S$. macrogoltabidus and that $S$. mali does not have SGL-1'.

On the standpoint of toxicity and environmental pollution, DD is one of the most important toxic substances for all life on Earth. Strain $\mathrm{RW} 1^{\mathrm{T}}$ is thus far a single strain of a new species, for which the name Sphingomonas wittichii sp. nov. is herein proposed. It is expected that by establishing the nomenclatural standing of this organism, research work on dioxin-degrading bacteria will be accelerated.

\section{Description of Sphingomonas wittichi sp. nov.}

Sphingomonas wittichii (wi.tti.chi.i. L. gen. n. wittichii of Wittich, referring to Rolf-Michael Wittich, the German bacteriologist who first isolated this potent metabolizer of dibenzo- $p$-dioxin from the water of the River Elbe and described the metabolism of dibenzo- $p$ dioxin by this organism).

Colonies are pin-point after $2 \mathrm{~d}$ incubation and, after a further $2-3 \mathrm{~d}$ are $1 \mathrm{~mm}$ in diameter. They are smooth, shiny, convex, semi-translucent and initially greyishwhite, becoming faintly yellow after $3 \mathrm{~d}$ incubation at $30^{\circ} \mathrm{C}$. Gram-negative rods with rounded ends. Soma size is $0.4-0.6 \times 0.9-1.5 \mu \mathrm{m}$. Actively motile with single polar or subpolar flagellum. Flagellar wave morphology is regular (Fig. 2a, b) or with only one long curve (Fig. 2c). Able to grow at $30^{\circ} \mathrm{C}$. Oxidaseand catalase-positive. Metabolism is respiratory, never 
Table 8 Phenotypic characteristics for differentiating Sphingomonas sp. strain RW1 $1^{\top}$ from the type strains of four other Sphingomonas species

+ , Positive reaction; -, negative reaction; $\mathrm{R}$, resistant; $\mathrm{S}$, susceptible.

\begin{tabular}{|c|c|c|c|c|c|}
\hline Characteristic & $\begin{array}{l}\text { Strain RW1 } \\
(=\text { EY 4224 } \\
(=1 T)\end{array}$ & $\begin{array}{l}\text { S. yanoikuyae } \\
{\text { EY } 4208^{\mathrm{T}}}^{\text {Th }}\end{array}$ & $\begin{array}{l}\text { S. terrae } \\
\text { EY } 4207^{\mathrm{T}}\end{array}$ & $\begin{array}{l}\text { S. macrogoltabidus } \\
\text { EY } 4304^{\mathrm{T}}\end{array}$ & $\begin{array}{c}\text { S. paucimobilis } \\
\text { EY } 2395^{\mathrm{T} *}\end{array}$ \\
\hline Galacturonosyl ceramide & + & + & + & + & - \\
\hline Deep yellow pigmentation & - & - & - & - & - \\
\hline Utilization of citrate (Simmons) & - & + & + & - & - \\
\hline Hydrolysis of gelatin & + & - & + & - & - \\
\hline DNase & - & + & + & - & + \\
\hline \multicolumn{6}{|l|}{ Acid from: } \\
\hline Inulin & + & - & - & + & - \\
\hline Sorbitol, mannitol & + & - & + & - & - \\
\hline \multicolumn{6}{|l|}{ Assimilation of: } \\
\hline Malonate & + & - & - & - & - \\
\hline Caprate & - & + & - & - & - \\
\hline DL- $\alpha$-Amino-n-butyrate & - & - & - & + & - \\
\hline $\mathrm{D}(-)$ Tartrate & - & - & + & - & - \\
\hline \multicolumn{6}{|l|}{ Susceptibility to: } \\
\hline Penicillin & $\mathrm{S}$ & $\mathrm{S}$ & $\mathrm{S}$ & $\mathrm{R}$ & $\mathrm{S}$ \\
\hline Piperacillin & $\mathrm{S}$ & $\mathrm{R}$ & $\mathrm{S}$ & $\mathrm{R}$ & $\mathrm{R}$ \\
\hline Cefmetazole & $\mathrm{S}$ & $\mathrm{R}$ & $\mathrm{R}$ & $\mathrm{R}$ & $\mathrm{R}$ \\
\hline Clarisharomycin & $\mathrm{R}$ & $\mathrm{S}$ & $\mathrm{R}$ & $\mathrm{S}$ & $\mathrm{S}$ \\
\hline Ofloxacin, sparfloxacin & $\mathrm{R}$ & $\mathrm{S}$ & $\mathrm{S}$ & $\mathrm{S}$ & $S$ \\
\hline
\end{tabular}

* Type species of the genus.

fermentative or photosynthetic. Hydrolyses aesculin, liquefies gelatin and alkalinizes malonate broth, but does not alkalinize Simmons' citrate medium and does not hydrolyse starch or Tween 80 . Failed to grow on MacConkey agar medium. The following tests are negative: deoxyribonuclease, nitrate reduction to nitrite, acylamidase, phenylalanine deaminase, lysine and ornithine decarboxylase and arginine dihydrolase. In OF medium, oxidative acidity observed from Darabinose, D-ribose, fructose, galactose, glucose, maltose, glycerol, sorbitol, mannitol, rhamnose, salicin and inulin within $3 \mathrm{~d}$. Among the 99 substrates of Biotype 100 (bioMérieux), only two carbohydrates, $\alpha-\mathrm{L}(-)$ fucose and $\mathrm{D}$-glucuronate are assimilated; the amino acids phenylalanine, L-glutamate, L-proline, Dalanine, $\mathrm{L}$-alanine, L-aspartate and $\mathrm{L}$-tyrosine and the organic acids gentisate, DL-lactate, succinate, fumarate, DL- $\beta$-hydroxybutyrate and malonate are assimilated. Resistant to macrolide and new quinolone antibiotics. Major fatty acid of cellular lipid is $18: 1$; the only hydroxy acid is 2-OH 14:0. Alkali-stable cellular lipids contain both SGL-1 and SGL-1'. DNA $\mathrm{G}+\mathrm{C}$ content is $67 \mathrm{~mol} \%$ (Wittich et al., 1992). Type strain is DSM $6014^{\mathrm{T}}\left(=\mathrm{JCM} 10273^{\mathrm{T}}=\mathrm{EY} 4224^{\mathrm{T}}\right)$.

\section{ACKNOWLEDGEMENTS}

A part of this work was supported by grants from Research on Environmental Health, H11-Saikatsu-015, and Research on Emerging and Re-emerging Infectious Diseases
(Ministry of Health and Welfare, Japan) and the US-Japan Cooperative Medical Science Program against Tuberculosis and Leprosy.

\section{REFERENCES}

Armengaud, J. \& Timmis, K. N. (1997). Molecular characterization of $\mathrm{Fdx} 1$, a putidaredoxin-type [2Fe-2S] ferredoxin able to transfer electrons to the dioxin dioxygenase of Sphingomonas sp. RW1. Eur J Biochem 247, 833-842.

Armengaud, J., Happe, B. \& Timmis, K. N. (1998). Genetic analysis of dioxin dioxygenase of Sphingomonas sp. strain RW1: catabolic genes dispersed on the genome. J Bacteriol 180, 3954-3966.

Bünz, P. V. \& Cook, A. M. (1993). Dibenzofuran 4,4 $\alpha$-dioxygenase from Sphingomonas sp. strain RW1: angular dioxygenation by a three-component enzyme system. J Bacteriol 175, 6467-6475.

Bünz, P. V., Falchetto, R. \& Cook, A. M. (1993). Purification of two isofunctional hydrolases (EC 3.7.1.8) in the degradative pathway for dibenzofuran in Sphingomonas sp. strain RW1. Biodegradation 4, 171-178.

Dagher, F., Deziel, E., Lirette, P., Paquette, G., Bisaillon, J.-G. \& Villemur, R. (1997). Comparative study of five polycyclic aromatic hydrocarbon degrading bacterial strains isolated from contaminated soils. Can J Microbiol 43, 368-377.

Davison, A. D., Karuso, P., Jardine, D. R. \& Veal, D. A. (1996). Halopicolinic acids, novel products arising through the degradation of chloro- and bromo-biphenyl by Sphingomonas paucimobilis BPSI-3. Can J Microbiol 42, 66-71.

Ezaki, T., Hashimoto, Y. \& Yabuuchi, E. (1989). Fluorometric DNA-DNA hybridization in microdilution wells as an alterna- 
tive to membrane filter hybridization in which radioisotopes are used to determine genetic relatedness among bacterial strains. Int J Syst Bacteriol 39, 224-229.

Happe, B., Eltis, L. D., Poth, H., Hedderich, R. \& Timmis, K. N. (1993). Characterization of 2,2'3-trihydroxybiphenyl dioxygenase, an extradiol dioxygenase from the dibenzofuran- and dibenzo-p-dioxin-degrading bacterium. Sphingomonas sp. strain RW1. J Bacteriol 175, 7313-7320.

Holmes, B., Owen, R. J., Evans, A., Malnick, H. \& Wilcox, W. R. (1977). Pseudomonas paucimobilis, a new species isolated from human clinical specimens, the hospital environment, and other sources. Int J Syst Bacteriol 27, 133-146.

Karlson, U., Rojo, F., Van Elsas, J. D. \& Moore, E. (1996). Genetic and serological evidence for the recognition of four pentachlorophenol-degrading bacterial strains as a species of the genus Sphingomonas. Syst Appl Microbiol 18, 539-548.

Kimura, M. (1980). A simple method for estimating evolutionary rates of base substitutions through comparative studies of nucleic acid sequences. $J$ Mol Evol 16, 111-120.

Khan, A. A., Wang, R.-F., Cao, W.-W., Franklin, W. \& Cerniglia, C. E. (1996). Reclassification of a polycyclic aromatic hydrocarbon-metabolizing bacterium, Beijerinckia sp. strain B1, as Sphingomonas yanoikuyae by fatty acid analysis, protein pattern analysis, DNA-DNA hybridization, and 16S ribosomal DNA sequencing. Int $J$ Syst Bacteriol 46, 466-469.

Kusunoki, S., Ezaki, T., Tamesada, M., Hatanaka, Y., Asano, K., Hashimoto, Y. \& Yabuuchi, E. (1991). Application of colorimetric microdilution plate hybridization for rapid genetic identification of 22 mycobacterium species. J Clin Microbiol 29, 1596-1603.

McCarthy, D. L., Navarrete, S., Willen, W. S., Babbitt, P. C. \& Copley, S. D. (1996). Exploration of the relationship between tetrachlorohydroquinone dehalogenase and the glutathione $S$ transferase superfamily. Biochemistry 35, 14634-14642.

Masai, E., Shinohara, S., Hara, H., Nishikawa, S., Katayama, Y. \& Fukuda, M. (1999). Genetic and biochemical characterization of a 2-pyrone-4,6-dicarboxylic acid hydrolase involved in the protocatechuate 4,5-cleavage pathway of Sphingomonas paucimobilis SYK-6. J Bacteriol 181, 55-62.

Megharaj, M., Wittich, R.-M., Blasco, R., Pieper, D. H. \& Timmis, K. N. (1997). Superior survival and degradation of dibenzo-pdioxin and dibenzofuran in soil by soil-adapted Sphingomonas sp. strain RW1. Appl Microbiol Biotechnol 48, 109-114.

Miyauchi, K., Suh, S. K., Nagata, Y. \& Takagi, M. (1998). Cloning and sequencing of a 2,5-dichlorohydroquinone reductive dehalogenase gene whose product is involved in degradation of gamma-hexachlorocyclohexamine by Sphingomonas paucimobilis. J Bacteriol 180, 1354-1359.

Naka, T., Fujiwara, N., Yabuuchi, E., Doe, M., Kobayashi, K., Kato, Y. \& Yano, I. (2000). A novel sphingoglycolipid containing galacturonic acid and 2-hydroxy fatty acid in cellular lipids of Sphingomonas yanoikuyae. J Bacteriol 182, 2660-2663.

Nickel, K., Suter, M. J.-F. \& Kohler, H.-P. (1997). Involvement of two alpha-ketoglutarate-dependent dioxygenases in enantioselective degradation of (R)- and (S)-mecoprop by Sphingomonas herbicidovorans MH. J Bacteriol 179, 6674-6679.

Nohynek, L., Suhonen, E. L., Nurmiaho-Lassila, E.-L., Hantula, J. \& Salkinoja-Salonen, M. (1995). Description of four pentachlorophenol-degrading bacterial strains as Sphingomonas chlorophenolica sp. nov. Syst Appl Microbiol 18, 527-538.

Peng, X., Egashira, T., Hanashiro, K., Masai, E., Nishikawa, S., Katayama, Y., Kimbara, K. \& Fukuda, M. (1998). A cloning of a
Sphingomonas paucimobilis SYK-6 gene encoding a novel oxygenase that cleaves lignin-related biphenyl and characterization of the enzyme. Appl Environ Microbiol 64, 2520-2527.

Romine, M. F., Stillwell, L. C., Wong, K. K., Thurston, S. J., Sisk, E. C., Sensoen, C., Gasterland, T., Freidrickson, J. K. \& Saffer, J. D. (1999). Complete sequence of a 184-kilobase catabolic plasmid from Sphingomonas aromaticivorans F199. J Bacteriol 181, 1585-1602.

Saitou, N. \& Nei, M. (1987). The neighbor-joining method: a new method for reconstructing phylogenetic trees. Mol Biol Evol 4, 406-425.

Schmidt, S., Wittich, R.-M., Erdmann, D., Wilkes, H., Francke, W. \& Fortnagel, P. (1992). Biodegradation of diphenyl ether and its monohalogenated derivatives by Sphingomonas sp. strain SS3. Appl Environ Microbiol 58, 2744-2750.

Schmidt, S., Fortnagel, P. \& Wittich, R.-M. (1993). Biodegradation and transformation of 4,4'- and 2,4-dihalodiphenyl ethers by Sphingomonas sp. strain SS33. Appl Environ Microbiol 59, 3931-3933.

Strubel, V., Rast, H. G., Fietz, W., Knackmuss, H.-J. \& Engesser, K. H. (1989). Enrichment of dibenzofuran utilizing bacteria with high co-metabolic potential towards dibenzodioxin and other anellated aromatics. FEMS Microbiol Lett 58, 233-238.

Takeuchi, M., Kawai, F., Shimada, Y. \& Yokota, A. (1993). Taxonomic study of polyethylene glycol-utilizing bacteria: emended description of the genus Sphingomonas and new descriptions of Sphingomonas macrogoltabidus sp. nov., Sphingomonas sanguis sp. nov. and Sphingomonas terrae sp. nov. Syst Appl Microbiol 16, 227-238.

Thomas, J.-C., Berger, F., Jacquier, M., Bernillon, D., Baud-Grasset, F., Truffaut, N., Normand, P., Vogel, T. M. \& Simonet, P. (1996). Isolation and characterization of a novel gamma-hexachlorocyclohexane-degrading bacterium. $J$ Bacteriol 178, 6049-6055.

Thompson, J. D., Higgins, D. G. \& Gibson, T. J. (1994). CLUSTAL $\mathrm{W}$ : improving the sensitivity of progressive multiple sequence alignment through sequence weighting, position-specific gap penalties and weight matrix choice. Nucleic Acids Res 22, 4673-4680.

Wilkes, H., Wittich, R. M., Timmis, K. N., Fortnagel, P. \& Francke, W. (1996). Degradation of chlorinated dibenzofurans and dibenzo-p-dioxins by Sphingomonas sp. strain RW1. Appl Environ Microbiol 62, 367-371.

Wittich, R.-M., Wilkes, H., Sinnwell, V., Francke, W. \& Fortnagel, P. (1992). Metabolism of dibenzo-p-dioxin by Sphingomonas sp. strain RW1. Appl Environ Microbiol 58, 1005-1010.

Yabuuchi, E., Yano, I., Oyaizu, H., Hashimoto, Y., Ezaki, T. \& Yamamoto, H. (1990). Proposals of Sphingomonas paucimobilis gen. nov. and comb. nov., Sphingomonas parapaucimobilis sp. nov., Sphingomonas yanoikuyae sp. nov., Sphingomonas adhaesiva sp. nov., Sphingomonas capsulata comb. nov., and two genospecies of the genus Sphingomonas. Microbiol Immunol 34, 99-119.

Yabuuchi, E., Kosako, Y., Naka, T., Suzuki, S. \& Yano, I. (1999). Proposal of Sphingomonas suberifaciens (van Bruggen, Jochimsen and Brown 1990) comb. nov., Sphingomonas natatoria (Sly 1985) comb. nov., Sphingomonas ursincola (Yurkov et al., 1997) comb. nov., and emendation of the genus Sphingomonas. Microbiol Immunol 43, 339-349.

Yrjala, K., Paulin, L. \& Romantschuk, M. (1997). Novel organization of catechol meta-pathway genes in Sphingomonas sp. HV3 pSKY4 plasmid. FEMS Microbiol Lett 154, 403-408. 
Yrjala, K., Suomalainen, S., Suhonen, E. L., Kilpi, S., Paulin, L. \& Romantschuk, M. (1998). Characterization and reclassification of an aromatic-and chloroaromatic-degrading Pseudomonas sp., strain HV3, as Sphingomonas sp. HV3. Int J Syst Bacteriol 48, 1057-1062.
Zipper, C., Nickel, K., Angst, W. \& Fohler, H.-P. E. (1996). Complete microbial degradation of both enantiomers of the chiral herbicide mecoprop [(RS)-2-(4-chloro-2-methylphenoxy) propionic acid] in an enantioselective manner by Sphingomonas herbicidovorans sp. nov. Appl Environ Microbiol 62, 4318-4322. 